\title{
THE THRESHOLD EFFECTS FOR THE TWO-PARTICLE HAMILTONIANS ON LATTICES
}

\author{
S. ALBEVERIO ${ }^{1,2,3}$, S. N. LAKAEV ${ }^{4,6}$, K. A. MAKAROV ${ }^{5}$, AND Z. I. MUMINOV 6
}

\begin{abstract}
For a wide class of two-body energy operators $h(k)$ on the three-dimensional lattice $\mathbb{Z}^{3}, k$ being the two-particle quasi-momentum, we prove that if the following two assumptions (i) and (ii) are satisfied, then for all nontrivial values $k, k \neq 0$, the discrete spectrum of $h(k)$ below its threshold is non-empty. The assumptions are: (i) the twoparticle Hamiltonian $h(0)$ corresponding to the zero value of the quasi-momentum has either an eigenvalue or a virtual level at the bottom of its essential spectrum and (ii) the oneparticle free Hamiltonians in the coordinate representation generate positivity preserving semi-groups.
\end{abstract}

\section{INTRODUCTION}

The main goal of the present paper is to give a thorough mathematical treatment of the spectral properties for the two-particle lattice Hamiltonians with emphasis on new threshold phenomena that are not present in the continuous case (see, e.g., [4], [8], [13]-[15], [17] for relevant discussions and [9], [11], [16], [29] for the general study of the low-lying excitation spectrum for quantum systems on lattices).

The kinematics of quantum quasi-particles on lattices, even in the two-particle sector, is rather exotic. For instance, due to the fact that the discrete analogue of the Laplacian or its generalizations (see (2.1) and 4.1) are not rotationally invariant, the Hamiltonian of a system does not separate into two parts, one relating to the center-of-mass motion and the other one to the internal degrees of freedom. In particular, such a handy characteristics of inertia as mass is not available. Moreover, such a natural local substituter as the effective mass-tensor (of a ground state) depends on the quasi-momentum of the system and, in addition, it is only semi-additive (with respect to the partial order on the set of positive definite matrices). This is the so-called excess mass phenomenon for lattice systems (see, e.g., [15] and [17]): the effective mass of the bound state of an $N$-particle system is greater than (but, in general, not equal to) the sum of the effective masses of the constituent quasiparticles.

The two-particle problem on lattices, in contrast to the continuous case where the usual split-off of the center of mass can be performed, can be reduced to an effective one-particle problem by using the Gelfand transform instead: the underlying Hilbert space $\ell^{2}\left(\left(\mathbb{Z}^{3}\right)^{2}\right)$ is decomposed as a direct von Neumann integral associated with the representation of the discrete group $\mathbb{Z}^{3}$ by shift operators on the lattice and then, the total two-body Hamiltonian appears to be decomposable as well. In contrast to the continuous case, the corresponding fiber Hamiltonians $h(k)$ associated with the direct decomposition depend parametrically on the internal binding $k$, the quasi-momentum, which ranges over a cell of the dual lattice.

1991 Mathematics Subject Classification. Primary: 81Q10, Secondary: 35P20, 47N50.

Key words and phrases. Discrete Schrödinger operators, quantum mechanical two-particle systems, Hamiltonians, conditionally negative definite functions, dispersion relations, virtual level, eigenvalues, lattice. 
As a consequence, due to the loss of the spherical symmetry of the problem, the spectra of the family $h(k)$ turn out to be rather sensitive to the variation of the quasi-momentum $k$.

We recall that in the case of continuous Schrödinger operators one observes the emission of negative bound states from the continuous spectrum at so-called critical potential strength (see, e.g., [1], [14], [19], [26]). This phenomenon is closely related to the existence of generalized eigenfunctions, which are solutions of the Schrödinger equation with zero energy decreasing at infinity, but are not square integrable. These solutions are usually called zero-energy resonance functions and, in this case, the Hamiltonian is called a critical one and the Schrödinger operator is said to have a zero-energy resonance (virtual level). The appearance of negative bound states for critical (non-negative) Schrödinger operators under infinitesimally small negative perturbations is especially remarkable: it is the presence of zero-energy resonances in at least two of the two-particle subsystems that leads to the existence of infinitely many bound states for the corresponding three-body system, the Efimov effect (see, e.g., [2], [13], [18], [23], [24], [25] and [27]).

It turns out that in the two-body lattice case there exists an extra mechanism for the bound state(s) to emerge from the threshold of the critical Hamiltonians which has nothing to do with additional (effectively negative) perturbations of the potential term. The role of the latter is rather played by the adequate change of the kinetic term which is due to the nontrivial dependence of the fiber Hamiltonians $h(k)$ on the quasi-momentum $k$ and is related to the excess mass phenomenon for lattice systems mentioned above.

The main result of the paper, Theorem 5.4 is the (variational) proof of existence of the discrete spectrum below the bottom of the essential spectrum of the fiber Hamiltonians $h(k)$ for all non-zero values of the quasi-momentum $0 \neq k \in \mathbb{T}^{3}$, provided that the Hamiltonian $h(0)$ has either a virtual level or a threshold eigenvalue.

Apart from some technical smoothness assumptions upon the dispersion relation of normal modes $\varepsilon_{\alpha}(p)$, characterizing the free particles $\alpha=1,2$, and as well as on smoothness assumptions (in the momentum representation) on the two-particle interactions (Hypothesis 3.1 the only additional assumption made (Hypothesis 5.1) is that the one-particle free Hamiltonians (in the coordinate representation) generate positivity preserving semi-groups $\exp \left(-t \hat{h}_{\alpha}^{0}\right), t>0, \quad \alpha=1,2$. We remark that this property is automatically fulfilled for the standard Laplacian (discrete or continuous).

The paper is organized as follows.

In Section 2 we formulate the main hypotheses on the one-particle lattice systems and prove the basic inequality (see Lemma 2.2 below) for the dispersion relations that are conditionally negative definite. In Section 3 we introduce the concept of a virtual level for the lattice one-particle Hamiltonians and develop the necessary background for our further considerations. In Section 4 we describe the two-particle Hamiltonians in both the coordinate and the momentum representation, introduce the two-particle quasi-momentum, and decompose the energy operator into the von Neumann direct integral of the fiber Hamiltonians $h(k)$, thus providing the reduction to the effective one-particle case.

In Section 5 we obtain efficient bounds on the location of the discrete spectrum for the two-particle fiber Hamiltonian and prove the main result of this paper, Theorem 5.4 in the case where $h(0)$ has either a threshold eigenvalue or virtual level at the bottom of its essential spectrum.

In Appendix A for readers convenience, we give a proof of Proposition 3.9 which is a "lattice" analogue of a result due to Yafaev [28] in the continuous case. 
In Appendix B we construct an explicit example of a one-particle discrete Schrödinger operator on the three-dimensional lattice $\mathbb{Z}^{3}$ that possesses both a virtual level and threshold eigenvalue at the bottom of its essential spectrum (cf., e.g., [1], [3], and [12] for related discussions in the case of continuous Schrödinger operators).

\section{THE ONE-PARTICLE HAMILTONIAN}

2.1. Dispersion relations. The free Hamiltonian $\hat{h}^{0}$ of a quantum particle on the threedimensional lattice $\mathbb{Z}^{3}$ is usually associated with the following self-adjoint (bounded) multidimensional Toeplitz-type operator on the Hilbert space $\ell^{2}\left(\mathbb{Z}^{3}\right)$ (see, e.g., [15]):

$$
\left(\hat{h}^{0} \hat{\psi}\right)(x)=\sum_{s \in \mathbb{Z}^{3}} \hat{\varepsilon}(s) \hat{\psi}(x+s), \quad \hat{\psi} \in \ell^{2}\left(\mathbb{Z}^{3}\right) .
$$

Here the series $\sum_{s \in \mathbb{Z}^{3}} \hat{\varepsilon}(s)$ is assumed to be absolutely convergent, that is,

$$
\{\hat{\varepsilon}(s)\}_{s \in \mathbb{Z}^{3}} \in \ell^{1}\left(\mathbb{Z}^{3}\right) .
$$

We also assume that the "self-adjointness" property is fulfilled

$$
\hat{\varepsilon}(s)=\overline{\hat{\varepsilon}(-s)}, \quad s \in \mathbb{Z}^{3} .
$$

In the physical literature, the symbol of the Toeplitz operator $\hat{h}^{0}$ given by the Fourier series

$$
\varepsilon(p)=\sum_{s \in \mathbb{Z}^{3}} \hat{\varepsilon}(s) e^{\mathrm{i}(p, s)}, \quad p \in \mathbb{T}^{3},
$$

being a real valued-function on $\mathbb{T}^{3}$, is called the dispersion relations of normal modes associated with the free particle in question. The one-particle free Hamiltonian is required to be of the form

$$
\hat{h}^{0}=\varepsilon(-\mathrm{i} \nabla),
$$

where $\nabla$ is the generator of the infinitesimal translations.

Under the mild assumption that

$$
\hat{v} \in \ell^{\infty}\left(\mathbb{Z}^{3}\right),
$$

where $\hat{v}=\{\hat{v}(s)\}_{s \in \mathbb{Z}^{3}}$ is a sequence of reals, the one-particle Hamiltonian $\hat{h}$,

$$
\hat{h}=\hat{h}^{0}+\hat{v},
$$

describing the quantum particle moving in the potential field $\hat{v}$, is a bounded self-adjoint operator on the Hilbert space $\ell^{2}\left(\mathbb{Z}^{3}\right)$.

The one-particle Hamiltonian $h$ in the momentum representation is introduced as

$$
h=\mathcal{F}^{-1} \hat{h} \mathcal{F},
$$

where $\mathcal{F}$ stands for the standard Fourier transform $\mathcal{F}: L^{2}\left(\mathbb{T}^{3}\right) \longrightarrow \ell^{2}\left(\mathbb{Z}^{3}\right)$, and $\mathbb{T}^{3}$ denotes the three-dimensional torus, the cube $(-\pi, \pi]^{3}$ with appropriately identified sides. Throughout the paper the torus $\mathbb{T}^{3}$ will always be considered as an abelian group with respect to the addition and multiplication by real numbers regarded as operations on $\mathbb{R}^{3}$ modulo $(2 \pi \mathbb{Z})^{3}$. 
2.2. Hamiltonians generating the positivity preserving semi-groups. The following important subclass of the one-particle systems is of certain interest (see, e.g., [6]). It is introduced by the additional requirement that the dispersion relation $\varepsilon(p)$ is a realvalued continuous conditionally negative definite function. Recall (see, e.g., [21]) that a complex-valued bounded function $\varepsilon: \mathbb{T}^{m} \longrightarrow \mathbb{R}$ is called conditionally negative definite if $\varepsilon(p)=\overline{\varepsilon(-p)}$ and

$$
\sum_{i, j=1}^{n} \varepsilon\left(p_{i}-p_{j}\right) z_{i} \bar{z}_{j} \leq 0
$$

for all $p_{1}, p_{2}, . ., p_{n} \in \mathbb{T}^{m}$ and all $\mathbf{z}=\left(z_{1}, z_{2}, \ldots, z_{n}\right) \in \mathbb{C}^{n}$ satisfying $\sum_{i=1}^{n} z_{i}=0$.

It is known that in this case the dispersion relation $\varepsilon(p)$ admits the (Lévy-Khinchin) representation (see, e.g., [5])

$$
\varepsilon(p)=\varepsilon(0)+\sum_{s \in \mathbb{Z}^{3} \backslash\{0\}}\left(e^{\mathrm{i}(p, s)}-1\right) \hat{\varepsilon}(s), \quad p \in \mathbb{T}^{3},
$$

which is equivalent to the requirement that the Fourier coefficients $\hat{\varepsilon}(s)$ with $s \neq 0$ are non-positive, that is,

$$
\hat{\varepsilon}(s) \leq 0, \quad s \neq 0,
$$

and the series $\sum_{s \in \mathbb{Z}^{3} \backslash\{0\}} \hat{\varepsilon}(s)$ converges absolutely. In turn, this is also equivalent to that the lattice Hamiltonian $\hat{h}=\hat{h}^{0}+v$ generates the positivity preserving semi-group $e^{-t \hat{h}}$, $t>0$, on $\ell^{2}\left(\mathbb{Z}^{3}\right)$ (see, e.g., [21] Ch. XIII). Following [6] we call the free Hamiltonians $\hat{h}^{0}=\varepsilon(-\mathrm{i} \nabla)$ generating the positivity preserving semi-groups the generalized Laplacians.

The following example shows that the standard discrete Laplacian is a generalized Laplacian in the sense mentioned above.

Example 2.1. For the one-particle free Hamiltonian

$$
\left(\hat{h}^{0} \hat{\psi}\right)(x)=(-\Delta \hat{\psi})(x)=\sum_{|s|=1}[\hat{\psi}(x)-\hat{\psi}(x+s)], \quad x \in \mathbb{Z}^{3}, \quad \hat{\psi} \in \ell^{2}\left(\mathbb{Z}^{3}\right),
$$

the (Fourier) coefficients $\hat{\varepsilon}(s), s \in \mathbb{Z}^{3}$, from 2.1) are necessarily of the form

$$
\hat{\varepsilon}(s)= \begin{cases}6, & s=0 \\ -1, & |s|=1 \\ 0, & \text { otherwise } .\end{cases}
$$

Hence, the corresponding dispersion relation

$$
\varepsilon(p)=2 \sum_{i=1}^{3}\left(1-\cos p_{i}\right), \quad p=\left(p_{1}, p_{2}, p_{3}\right) \in \mathbb{T}^{3},
$$

is a conditionally negative definite function.

We need a simple inequality which will play a crucial role in the proof of the main results of the paper, Theorems 5.3 and 5.4

Lemma 2.2. Assume that the dispersion relation $\varepsilon(p)$ is a real-valued continuous conditionally negative definite function on $\mathbb{T}^{3}$. Assume, in addition, that $\varepsilon(0)$ is the unique minimum of the function $\varepsilon(p)$. Then for all $q \in \mathbb{T}^{3} \backslash\{0\}$ the inequality

$$
\varepsilon(p)+\varepsilon(q)>\frac{\varepsilon(p+q)+\varepsilon(p-q)}{2}+\varepsilon(0), \quad \text { a.e. } \quad p \in \mathbb{T}^{3},
$$

holds. 
Proof. For $n=4, m=3$,

$$
\begin{aligned}
& p_{1}=\frac{p+q}{2}, \\
& p_{3}=-p_{1}, \quad p_{2}=\frac{q-p}{2},
\end{aligned}
$$

and $\mathbf{z}=(1,-1,1,-1)$ applying inequality $(2.2)(\varepsilon(p)$ is an even function) proves the non-strict version of inequality 2.4. To show that inequality 2.4) is strict we proceed as follows.

Fix a $q \in \mathbb{T}^{3}, q \neq 0$. Then there exists an $s_{0} \in \mathbb{Z}^{3} \backslash\{0\}$ such that $\hat{\varepsilon}\left(s_{0}\right)<0$ and $\cos \left(q, s_{0}\right) \neq 1$ (otherwise $\varepsilon(q)=\sum_{s \in \mathbb{Z}^{3}} \hat{\varepsilon}(s)=\varepsilon(0)$ which contradicts the hypothesis that $\varepsilon(0)$ is the unique minimum of the function $\varepsilon(\cdot)$ on $\left.\mathbb{T}^{3}\right)$. Since the cosine function is conditionally positive definite, using the non-strict version of inequality (2.4) yields

$$
\begin{aligned}
F(p, q) & \equiv \varepsilon(p)+\varepsilon(q)-\frac{\varepsilon(p+q)+\varepsilon(p-q)}{2}-\varepsilon(0) \\
& =\sum_{s \in \mathbb{Z}^{3}} \hat{\varepsilon}(s)\left[\cos (p, s)+\cos (q, s)-\frac{\cos (p+q, s)+\cos (p-q, s)}{2}-1\right] \\
& \geq 2 \hat{\varepsilon}\left(s_{0}\right)\left[\cos \left(p, s_{0}\right)+\cos \left(q, s_{0}\right)-\frac{\cos \left(p+q, s_{0}\right)+\cos \left(p-q, s_{0}\right)}{2}-1\right] \\
& =2 \hat{\varepsilon}\left(s_{0}\right)\left[\cos \left(p, s_{0}\right)+\cos \left(q, s_{0}\right)-\cos \left(p, s_{0}\right) \cos \left(q, s_{0}\right)-1\right]
\end{aligned}
$$

Hence

$$
F(p, q) \geq 2 \hat{\varepsilon}\left(s_{0}\right)\left[\left(\cos \left(p, s_{0}\right)-1\right)\left(1-\cos \left(q, s_{0}\right)\right)\right]>0, \quad\left(p, s_{0}\right) \neq 2 n \pi, \quad n \in \mathbb{Z},
$$

since $\hat{\varepsilon}\left(s_{0}\right)<0$ and $\cos \left(q, s_{0}\right) \neq 1$, completing the proof.

\section{A ViRTuAl LEVEl AND THREShold EigenVAlues}

In order to introduce the concept of a virtual level (threshold resonance) for the (lattice) energy operator $h$ we assume the following technical hypotheses that guarantee some smoothness of the dispersion relation $\varepsilon(p)$ and the continuity of the Fourier transform

$$
v(p)=(2 \pi)^{-\frac{3}{2}} \sum_{s \in \mathbb{Z}^{3}} \hat{v}(s) e^{\mathrm{i}(p, s)}
$$

of the interaction $\hat{v}$.

Hypothesis 3.1. Assume that the dispersion relation $\varepsilon(p)$ is a twice differentiable (periodic) real-valued functions on $\mathbb{T}^{3}$ with a unique non-degenerate minimum at the origin. Assume, in addition, that $v(p)$ is a continuous function on $\mathbb{T}^{3}$ such that

$$
v(p)=\overline{v(-p)}, \quad p \in \mathbb{T}^{3} .
$$

For $\lambda \leq \varepsilon(0)$ on the Banach space $C\left(\mathbb{T}^{3}\right)$ of continuous (periodic) functions on $\mathbb{T}^{3}$ we shall consider the integral operator $G(\lambda)$ with the (Birman-Schwinger) kernel function

$$
G(p, q ; \lambda)=(2 \pi)^{-\frac{3}{2}} v(p-q)(\varepsilon(q)-\lambda)^{-1}, \quad p, q \in \mathbb{T}^{3} .
$$

Lemma 3.2. Assume Hypothesis 3.1] Then for $\lambda \leq \varepsilon(0)$ the operator $G(\lambda)$ on $C\left(\mathbb{T}^{3}\right)$ given by (3.1) is compact. 
Proof. Given $f \in L^{1}\left(\mathbb{T}^{3}\right)$, for the function $g$ introduced by

$$
g(p)=(2 \pi)^{-\frac{3}{2}} \int_{\mathbb{T}^{3}} v(p-q) f(q) d q
$$

one has the estimates

$$
|g(p)| \leq(2 \pi)^{-\frac{3}{2}} \sup _{p, q \in \mathbb{T}^{3}}|v(p-q)|\|f\|_{L^{1}\left(\mathbb{T}^{3}\right)}
$$

and

$$
\begin{gathered}
|g(p+\ell)-g(p)|=\left|(2 \pi)^{-\frac{3}{2}} \int_{\mathbb{T}^{3}}(v(p+\ell-q)-v(p-q)) f(q) d q\right| \\
\leq(2 \pi)^{-\frac{3}{2}} \sup _{t \in \mathbb{T}^{3}}|v(t+\ell)-v(t)|\|f\|_{L^{1}\left(\mathbb{T}^{3}\right)} .
\end{gathered}
$$

Since for $\lambda \leq \varepsilon(0)$, the function $(\varepsilon(\cdot)-\lambda)^{-1}$, is summable, the multiplication operator by the function $(\varepsilon(\cdot)-\lambda)^{-1}$ from $C\left(\mathbb{T}^{3}\right)$ into $L^{1}\left(\mathbb{T}^{3}\right)$ is continuous. Therefore, from 3.2 and 3.3 it follows that the image of the unit ball in $C\left(\mathbb{T}^{3}\right)$ consists of functions that are totally bounded and equicontinuous: $v$ is continuous and, therefore,

$$
\lim _{|\ell| \rightarrow 0} \sup _{t \in \mathbb{T}^{3}}|v(t+\ell)-v(t)|\|f\|_{L^{1}\left(\mathbb{T}^{3}\right)}=0 .
$$

An application of the Arzela-Ascoli Theorem then completes the proof.

Remark 3.3. Clearly (cf. [28]), the operator $h$ has an eigenvalue $\lambda \leq \varepsilon(0)$, that is, $\operatorname{Ker}(h-\lambda I) \neq 0$, if and only if the compact operator $G(\lambda)$ on $C\left(\mathbb{T}^{3}\right)$ has an eigenvalue -1 and there exists a function $\psi \in \operatorname{Ker}(G+I)$ such that the function $f$ given by

$$
f(p)=\frac{\psi(p)}{\varepsilon(p)-\lambda} \quad \text { a.e. } \quad p \in \mathbb{T}^{3},
$$

belongs to $L^{2}\left(\mathbb{T}^{3}\right)$. In this case $f \in \operatorname{Ker}(h-\lambda I)$.

Moreover, if $\lambda<\varepsilon(0)$, then

$$
\operatorname{dim} \operatorname{Ker}(h-\lambda I)=\operatorname{dim} \operatorname{Ker}(G(\lambda)+I)
$$

and

$$
\operatorname{Ker}(h-\lambda I)=\left\{f \mid f(\cdot)=\frac{\psi(\cdot)}{\varepsilon(\cdot)-\lambda}, \psi \in \operatorname{Ker}(G(\lambda)+I)\right\} .
$$

In the case of a threshold eigenvalue $\lambda=\varepsilon(0)$ equality (3.4 may fail to hold. It should be replaced by the inequality

$$
\operatorname{dim} \operatorname{Ker}(h-\varepsilon(0) I) \leq \operatorname{dim} \operatorname{Ker}(G(\varepsilon(0))+I) .
$$

In order to discuss the threshold phenomena, that is, the case $\lambda=\varepsilon(0)$, following [3] and [7] (see also [12] for a related discussion), under Hypothesis 3.1 we distinguish five mutually disjoint cases:

Case I: -1 is not an eigenvalue of $G(\varepsilon(0))$.

Case II: -1 is a simple eigenvalue of $G(\varepsilon(0))$ and the associated eigenfunction $\psi$ satisfies the condition

$$
\frac{\psi(\cdot)}{\varepsilon(\cdot)-\varepsilon(0)} \notin L^{2}\left(\mathbb{T}^{3}\right) .
$$


Case III: -1 is an eigenvalue of $G(\varepsilon(0))$ and any of the associated eigenfunctions $\psi$ satisfies the condition

$$
\frac{\psi(\cdot)}{\varepsilon(\cdot)-\varepsilon(0)} \in L^{2}\left(\mathbb{T}^{3}\right)
$$

Case IV: -1 is a multiple eigenvalue of $G(\varepsilon(0))$ and exactly one (up to a normalization) of the associated eigenfunctions $\psi$ satisfies the condition

$$
\frac{\psi(\cdot)}{\varepsilon(\cdot)-\varepsilon(0)} \notin L^{2}\left(\mathbb{T}^{3}\right) \text {. }
$$

Case V: -1 is a multiple eigenvalue of $G(\varepsilon(0))$ and at least two of the associated eigenfunctions $\psi$ and $\varphi$ that are linearly independent satisfy the condition

$$
\frac{\psi(\cdot)}{\varepsilon(\cdot)-\varepsilon(0)} \notin L^{2}\left(\mathbb{T}^{3}\right) \quad \text { and } \frac{\varphi(\cdot)}{\varepsilon(\cdot)-\varepsilon(0)} \notin L^{2}\left(\mathbb{T}^{3}\right) .
$$

Given the classification above, we arrive at the following definition of a virtual level.

Definition 3.4. In Cases II, IV and V the operator $h$ is said to have a virtual level (at the threshold).

Remark 3.5. Note that in Cases III and IV the operator $h$ has a threshold eigenvalue $\lambda=\varepsilon(0)$ of multiplicity $\operatorname{dim} \operatorname{Ker}(G(\varepsilon(0))+I)$ and $\operatorname{dim} \operatorname{Ker}(G(\varepsilon(0))+I)-1$ respectively.

Remark 3.6. Our definition of a virtual level is equivalent to the direct analogue of that in the continuous case (see, e.g., [1], [23], [25], [27], [28] and references therein).

Remark 3.7. If the Hamiltonian $h$ has a virtual level and the corresponding function $\psi$, $\psi \in \operatorname{Ker}(G(\varepsilon(0))+I)$, is such that $\frac{\psi(\cdot)}{\varepsilon(\cdot)-\varepsilon(0)} \notin L^{2}\left(\mathbb{T}^{3}\right)$, then the function

$$
f(p)=\frac{\psi(p)}{\varepsilon(p)-\varepsilon(0)}, \quad p \in \mathbb{T}^{3},
$$

belongs to $L^{r}\left(\mathbb{T}^{3}\right), 1 \leq r<3 / 2$.

In particular, the function $f$ given by (3.5) is the eigenfunction of the operator $h$ associated with the eigenvalue $\varepsilon(0)$ in the Banach space $L^{1}\left(\mathbb{T}^{3}\right)$, that is,

$$
h f=\varepsilon(0) f
$$

and hence the following equation

$$
\varepsilon(p) f(p)+(2 \pi)^{-\frac{3}{2}} \int_{\mathbb{T}^{3}} v(p-q) f(q) d q=\varepsilon(0) f(p), \quad \text { a.e. } p \in \mathbb{T}^{3},
$$

holds.

A simple computation shows that the Fourier coefficients $\hat{f}(s), s \in \mathbb{Z}^{3}$, of the (summable) function $f$ solve the infinite system of homogeneous equations

$$
\sum_{s \in \mathbb{Z}^{3}} \hat{\varepsilon}(s) \hat{f}(x+s)+(\hat{v}(x)-\varepsilon(0)) \hat{f}(x)=0, \quad x \in \mathbb{Z}^{3},
$$

and hence the equation (in the coordinate representation)

$$
\hat{h} \hat{f}=\varepsilon(0) \hat{f}
$$

has a solution $\hat{f}$, a threshold resonant state, that does not belong to $\ell^{2}\left(\mathbb{Z}^{3}\right)$ but vanishes at infinity,

$$
\lim _{|s| \rightarrow \infty} \hat{f}(s)=0
$$

(by the Riemann-Lebesgue Theorem). 
Remark 3.8. If the dispersion relation $\varepsilon(p)$ is known to be an even function, $\varepsilon(p)=\varepsilon(-p)$, or, which is the same, the Fourier coefficients satisfy the condition

$$
\hat{\varepsilon}(s)=\hat{\varepsilon}(-s) \in \mathbb{R}, \quad s \in \mathbb{Z}^{3},
$$

the Birman-Schwinger kernel $G(p, q ; \lambda)$ has the additional property that

$$
G(p, q ; \lambda)=\overline{G(-p,-q ; \lambda)} .
$$

Hence, if $\psi \in \operatorname{Ker}(G(\lambda)+I), \lambda \leq \varepsilon(0)$, so does the function $\varphi(p)=\overline{\psi(-p)}$. Therefore, exactly one of the functions $\psi \pm \varphi$ is also an eigenfunction of $G(\lambda)$ associated with the eigenvalue -1 , and hence, without loss of generality one may assume that the operator $G(\lambda)$ has an eigenfunction $\tilde{\psi}$ such that $|\tilde{\psi}(\cdot)|$ is an even function.

To get finer results (cf. [28]) we need an auxiliary scale of the Banach spaces $\mathcal{B}(\mu)$, $0<\mu \leq 1$, of Hölder continuous functions on $\mathbb{T}^{3}$ obtained by the closure of the space of smooth (periodic) functions $f$ on $\mathbb{T}^{3}$ with respect to the norm

$$
\|f\|_{\mu}=\sup _{t, \ell \in \mathbb{T}^{3}}\left[|f(t)|+|\ell|^{-\mu}|f(t+\ell)-f(t)|\right] .
$$

Note that the spaces $\mathcal{B}(\mu)$ are naturally embedded one into the other

$$
\mathcal{B}(\nu) \subset \mathcal{B}(\mu) \subset C\left(\mathbb{T}^{3}\right), \quad 0<\mu \leq \nu \leq 1 .
$$

If $v \in \mathcal{B}(\kappa)$ with $\kappa>\frac{1}{2}$, the following proposition, a variant of the Birman-Schwinger principle, is a convenient tool to decide whether the threshold $\varepsilon(0)$ of the essential spectrum of $h$ is an eigenvalue (resp. a virtual level ) for the operator $h$.

Proposition 3.9. (cf. [28]) Assume Hypotheses 3.1] Assume, in addition, that $v \in \mathcal{B}(\kappa)$ with $\kappa>\frac{1}{2}$. Then the operator $h(0)-\varepsilon(0) I$ has a non-trivial kernel if and only if -1 is an eigenvalue of $G(\varepsilon(0))$ and one of the associated eigenfunctions $\psi$ satisfies the condition

$$
\psi(0)=0 .
$$

In particular, the operator $h$ has a virtual level if and only if - 1 is an eigenvalue of $G(\varepsilon(0))$ and one of the associated eigenfunctions $\psi$ satisfies the condition

$$
\psi(0) \neq 0 \text {. }
$$

Proof. See Appendix

Remark 3.10. A more thorough examination of the proof shows that, in fact,

$$
\operatorname{dim} \operatorname{Ker}(h(0)-\varepsilon(0) I)= \begin{cases}\operatorname{dim} \operatorname{Ker}(G(\varepsilon(0))+I), & \text { in Case III } \\ \operatorname{dim} \operatorname{Ker}(G(\varepsilon(0))+I)-1, & \text { in Case IV. }\end{cases}
$$

Remark 3.11. If $\kappa>\frac{1}{2}$, as it follows from Proposition 3.9 the eigensubspace of functions $\psi$ associated with the eigenvalue $\lambda=-1$ of $G(\varepsilon(0))$ with the additional constraint $\psi(0) \neq$ 0 is one-dimensional. This proves that Case V does not occur if $\kappa>\frac{1}{2}$. In Case IV we have the coexistence of a (simple) virtual level and a (possibly multiple) threshold eigenvalue (see Appendix B for a concrete example of such a coexistence in Case IV).

Remark 3.12. It is known that for the continuous Schrödinger operators $h=-\Delta+V(x)$ with $V \in L^{1}\left(\mathbb{R}^{3}\right) \cap R, R$ the Rollnik class, Case V does not occur (see, [1] Lemma 1.2.3). It is also worth mentioning that if, in addition, the Schrödinger operator $h$ is non-negative, then under the $L^{\frac{3}{2}}$-weak assumption on the potential V Cases III, and IV do not occur (there is no zero-energy eigenstate) (see, e.g., [10], [22], and [24]). 


\section{The two-Particle Hamiltonian. Reduction to the OnE-PARTiCle CASE}

4.1. The coordinate representation. The free Hamiltonian $\widehat{H}^{0}$ of the system of two quantum particles $\alpha=1,2$, with the dispersion relations $\varepsilon_{\alpha}(p), \alpha=1,2$, respectively, is introduced (as a bounded self-adjoint operator on the Hilbert space $\ell^{2}\left(\left(\mathbb{Z}^{3}\right)^{2}\right) \simeq \ell^{2}\left(\mathbb{Z}^{3}\right) \otimes$ $\ell^{2}\left(\mathbb{Z}^{3}\right)$ ) by

$$
\widehat{H}^{0}=\hat{h}_{1}^{0} \otimes I+I \otimes \hat{h}_{2}^{0},
$$

with

$$
\hat{h}_{\alpha}^{0}=\varepsilon_{\alpha}(-\mathrm{i} \nabla), \quad \alpha=1,2,
$$

and $I$ the identity operator on $\ell^{2}\left(\mathbb{Z}^{3}\right)$.

The total Hamiltonian $\widehat{H}$ (in the coordinate representation) of the two-particle system with the real-valued pair interaction $\widehat{V}$ is a self-adjoint bounded operator on the Hilbert space $\ell^{2}\left(\left(\mathbb{Z}^{3}\right)^{2}\right)$ of the form

$$
\widehat{H}=\widehat{H}^{0}+\widehat{V}
$$

where

$$
(\widehat{V} \hat{\psi})\left(x_{1}, x_{2}\right)=\hat{v}\left(x_{1}-x_{2}\right) \hat{\psi}\left(x_{1}, x_{2}\right), \quad \hat{\psi} \in \ell^{2}\left(\left(\mathbb{Z}^{3}\right)^{2}\right),
$$

with $\{\hat{v}(s)\}_{s \in \mathbb{Z}^{3}}$ the Fourier coefficients of a continuous function $v(p)$ satisfying Hypothesis 3.1

4.2. The momentum representation. The transition to the momentum representation is performed by the standard Fourier transform

$$
\mathcal{F}_{2}: L^{2}\left(\left(\mathbb{T}^{3}\right)^{2}\right) \longrightarrow \ell^{2}\left(\left(\mathbb{Z}^{3}\right)^{2}\right),
$$

where $\left(\mathbb{T}^{3}\right)^{m}$ denotes the Cartesian $m$-th power of the three-dimensional cube $\mathbb{T}^{3}=$ $(-\pi, \pi]^{3}$ :

$$
\left(\mathbb{T}^{3}\right)^{m}=\underbrace{\mathbb{T}^{3} \times \mathbb{T}^{3} \times \cdots \times \mathbb{T}^{3}}_{m \text { times }}, \quad m \in \mathbb{N} .
$$

The two-particle Hamiltonian $H$ in the momentum representation is then given by

$$
H=H^{0}+V
$$

where

$$
\left(H^{0} f\right)\left(k_{1}, k_{2}\right)=\left(\varepsilon_{1}\left(k_{1}\right)+\varepsilon_{2}\left(k_{2}\right)\right) f\left(k_{1}, k_{2}\right), \quad f \in L^{2}\left(\left(\mathbb{T}^{3}\right)^{2}\right),
$$

and $V$ is the operator of partial integration given by

$$
\begin{gathered}
(V f)\left(k_{1}, k_{2}\right)=(2 \pi)^{-\frac{3}{2}} \int_{\left(\mathbb{T}^{3}\right)^{2}} v\left(k_{1}-k_{1}^{\prime}\right) \delta\left(k_{1}+k_{2}-k_{1}^{\prime}-k_{2}^{\prime}\right) f\left(k_{1}^{\prime}, k_{2}^{\prime}\right) d k_{1}^{\prime} d k_{2}^{\prime}, \\
f \in L^{2}\left(\left(\mathbb{T}^{3}\right)^{2}\right) .
\end{gathered}
$$

Here the kernel function is given by the Fourier series

$$
v(p)=(2 \pi)^{-3 / 2} \sum_{s \in \mathbb{Z}^{3}} \hat{v}(s) e^{\mathrm{i}(p, s)}, \quad p \in \mathbb{T}^{3},
$$

and $\delta(p)$ denotes the Dirac delta-function. 
4.3. Direct integral decompositions. The quasi-momentum. Denote by $\hat{U}_{s}^{2}, s \in \mathbb{Z}^{3}$, the unitary representation of the abelian group $\mathbb{Z}^{3}$ by the shift operators on the Hilbert space $\ell^{2}\left(\left(\mathbb{Z}^{3}\right)^{2}\right)$ :

$$
\left(\hat{U}_{s}^{2} \hat{\psi}\right)\left(n_{1}, n_{2}\right)=\hat{\psi}\left(n_{1}+s, n_{2}+s\right), \quad \hat{\psi} \in \ell^{2}\left(\left(\mathbb{Z}^{3}\right)^{2}\right), \quad n_{1}, n_{2}, s \in \mathbb{Z}^{3} .
$$

Via the Fourier transform $\mathcal{F}_{2}$ the unitary representation

$$
\hat{U}_{s+t}^{2}=\hat{U}_{s}^{2} \hat{U}_{t}^{2}, \quad s, t \in \mathbb{Z}^{3},
$$

induces the representation of the group $\mathbb{Z}^{3}$ in the Hilbert space $L^{2}\left(\left(\mathbb{T}^{3}\right)^{2}\right)$ by unitary (multiplication) operators $U_{s}^{2}=\mathcal{F}_{2}^{-1} \hat{U}_{s}^{2} \mathcal{F}_{2}, s \in \mathbb{Z}^{3}$,

(4.3) $\left(U_{s}^{2} f\right)\left(k_{1}, k_{2}\right)=\exp \left(-\mathrm{i}\left(s, k_{1}+k_{2}\right)\right) f\left(k_{1}, k_{2}\right), \quad k_{1}, k_{2} \in \mathbb{T}^{3}, \quad f \in L^{2}\left(\left(\mathbb{T}^{3}\right)^{2}\right)$.

Given $k \in \mathbb{T}^{3}$, we define $\mathbb{F}_{k}$ as follows

$$
\mathbb{F}_{k}=\left\{\left(k_{1}, k-k_{1}\right) \in\left(\mathbb{T}^{3}\right)^{2}: k_{1} \in \mathbb{T}^{3}, k-k_{1} \in \mathbb{T}^{3}\right\} .
$$

Introducing the mapping

$$
\pi:\left(\mathbb{T}^{3}\right)^{2} \rightarrow \mathbb{T}^{3}, \quad \pi\left(\left(k_{1}, k_{2}\right)\right)=k_{1},
$$

we denote by $\pi_{k}, k \in \mathbb{T}^{3}$, the restriction of $\pi$ to $\mathbb{F}_{k} \subset\left(\mathbb{T}^{3}\right)^{2}$, that is,

$$
\pi_{k}=\left.\pi\right|_{\mathbb{F}_{k}} .
$$

We remark that $\mathbb{F}_{k}, k \in \mathbb{T}^{3}$, is a three-dimensional manifold homeomorphic to $\mathbb{T}^{3}$.

The following lemma is evident.

Lemma 4.1. The mapping $\pi_{k}, k \in \mathbb{T}^{3}$, from $\mathbb{F}_{k} \subset\left(\mathbb{T}^{3}\right)^{2}$ onto $\mathbb{T}^{3}$ is bijective, with the inverse mapping given by

$$
\left(\pi_{k}\right)^{-1}(q)=(q, k-q) .
$$

Decomposing the Hilbert space $L^{2}\left(\left(\mathbb{T}^{3}\right)^{2}\right)$ into the direct integral

$$
L^{2}\left(\left(\mathbb{T}^{3}\right)^{2}\right)=\int_{k \in \mathbb{T}^{3}} \oplus L^{2}\left(\mathbb{F}_{k}\right) d k
$$

yields the corresponding decomposition of the unitary representation $U_{s}^{2}, s \in \mathbb{Z}^{3}$, into the direct integral

$$
U_{s}^{2}=\int_{k \in \mathbb{T}^{3}} \oplus U_{s}(k) d k
$$

with

$$
U_{s}(k)=e^{-\mathrm{i}(s, k)} I_{L^{2}\left(\mathbb{F}_{k}\right)}
$$

and $I_{L^{2}\left(\mathbb{F}_{k}\right)}$ the identity operator on the Hilbert space $L^{2}\left(\mathbb{F}_{k}\right)$.

The Hamiltonian $\widehat{H}$ (in the coordinate representation) obviously commutes with the group of translations, $\hat{U}_{s}^{2}, s \in \mathbb{Z}^{3}$, that is,

$$
\hat{U}_{s}^{2} \widehat{H}=\widehat{H} \hat{U}_{s}^{2}, \quad s \in \mathbb{Z}^{3} .
$$

So does the Hamiltonian $H$ (in the momentum representation) with respect to the group $U_{s}^{2}, s \in \mathbb{Z}^{3}$, given by 4.3. Hence, the operator $H$ can be decomposed into the direct integral

$$
H=\int_{k \in \mathbb{T}^{3}} \oplus \tilde{h}(k) d k
$$


associated with the decomposition

$$
L^{2}\left(\left(\mathbb{T}^{3}\right)^{2}\right)=\int_{k \in \mathbb{T}^{3}} \oplus L^{2}\left(\mathbb{F}_{k}\right) d k .
$$

In the physical literature the parameter $k, k \in \mathbb{T}^{3}$, is called the two-particle quasi-momentum and the corresponding operators $\tilde{h}(k), k \in \mathbb{T}^{3}$, are called the fiber operators.

4.4. The two-particle dispersion relations. The fiber operators $\tilde{h}(k), k \in \mathbb{T}^{3}$, from the decomposition (4.5) are unitarily equivalent to the operators $h(k), k \in \mathbb{T}^{3}$, of the form

$$
h(k)=h^{0}(k)+v,
$$

where

$$
\begin{gathered}
\left(h^{0}(k) f\right)(p)=\mathcal{E}_{k}(p) f(p), \\
(v f)(p)=(2 \pi)^{-\frac{3}{2}} \int_{\mathbb{T}^{3}} v(p-q) f(q) d q, \quad f \in L^{2}\left(\mathbb{T}^{3}\right)
\end{gathered}
$$

and the two-particle dispersion relations

$$
\mathcal{E}_{k}(p)=\varepsilon_{1}(p)+\varepsilon_{2}(k-p), \quad p \in \mathbb{T}^{3},
$$

parametrically depend on the quasi-momentum $k, k \in \mathbb{T}^{3}$.

The equivalence is given by the unitary operator $u_{k}: L^{2}\left(\mathbb{F}_{k}\right) \rightarrow L^{2}\left(\mathbb{T}^{3}\right), k \in \mathbb{T}^{3}$,

$$
u_{k} g=g \circ\left(\pi_{k}\right)^{-1} \text {, }
$$

with $\pi_{k}$ defined by 4.4 .

\section{SPECTRAL PROPERTIES OF THE FIBER OPERATORS $h(k)$}

As we have learned from the previous section, the two-particle Hamiltonian $H$ (up to unitary equivalence) can be decomposed into the direct integral

$$
H \simeq \int_{k \in \mathbb{T}^{3}} \oplus h(k) d k,
$$

where the fiber operators

$$
h(k)=h^{0}(k)+v
$$

can be considered as the one-particle Hamiltonians with the two-particle dispersion relations

$$
\mathcal{E}_{k}(p)=\varepsilon_{1}(p)+\varepsilon_{2}(k-p), \quad p \in \mathbb{T}^{3},
$$

with $\varepsilon_{\alpha}(p)$ the dispersion relations for the particles $\alpha=1,2$.

Under Hypothesis 3.1 the perturbation $v$ of the operator $h^{0}(k), k \in \mathbb{T}^{3}$, is a HilbertSchmidt operator and, therefore, in accordance with the Weyl Theorem the essential spectrum of the operator $h(k)$ fills in the following interval on the real axis:

$$
\sigma_{\mathrm{ess}}(h(k))=\left[\mathcal{E}_{\min }(k), \mathcal{E}_{\max }(k)\right],
$$

where

$$
\mathcal{E}_{\min }(k)=\min _{q \in \mathbb{T}^{3}} \mathcal{E}_{k}(q), \quad \mathcal{E}_{\max }(k)=\max _{q \in \mathbb{T}^{3}} \mathcal{E}_{k}(q) .
$$

If the dispersion relations in the one-particle sector are conditionally negative definite, then so is the two-particle dispersion relation $\varepsilon_{0}(p)$ corresponding to the zero-value of the quasi-momentum $k$. Hence, under these assumptions, the Hamiltonian $h(0)$ in the coordinate representation generates the positivity preserving semi-group $e^{-t h(0)}, t>0$ (which is not necessarily true for the fiber Hamiltonians $h(k)$ with $k \neq 0$ : the function $\mathcal{E}_{k}(p)$ may 
not be even, and hence, not conditionally negative definite). Although the two-particle dispersion relations are not necessarily conditionally negative definite for nontrivial values of the quasi-momentum, they still satisfy some useful inequality, Lemma 5.2 below, analogous to that in Lemma2.2 for the one-particle dispersion relations.

Hypothesis 5.1. Assume Hypothesis 3.1 Suppose that the dispersion relations $\varepsilon_{\alpha}(p)$, $\alpha=1,2$, in the one-particle sectors are conditionally negative definite.

Lemma 5.2. Assume Hypothesis 5.1 Then for any (fixed) $k, q \in \mathbb{T}^{3}$ such that either $k \neq q$ or $q \neq 0$

$$
\mathcal{E}_{0}(p)-\mathcal{E}_{0}(0)+\mathcal{E}_{k}(q)-\frac{\mathcal{E}_{k}(p+q)+\mathcal{E}_{k}(q-p)}{2}>0, \quad \text { a.e. } \quad p \in \mathbb{T}^{3} .
$$

In particular, if $k \neq 0$, and $p(k)$ is a (any) point where the function $\mathcal{E}_{k}(\cdot)$ attains its minimal value, that is,

$$
\mathcal{E}_{\min }(k)=\mathcal{E}_{k}(p(k)),
$$

the following inequality

$$
\varepsilon_{0}(p)-\mathcal{E}_{\min }(0)+\mathcal{E}_{\min }(k)-\frac{\mathcal{E}_{k}(p+p(k))+\mathcal{E}_{k}(p(k)-p)}{2}>0, \quad \text { a.e. } \quad p \in \mathbb{T}^{3},
$$

holds.

Proof. Since $|q|^{2}+|k-q|^{2} \neq 0$ the claim is an immediate consequence of Lemma 2.2 and definition 5.1 of the two-particle dispersion relations:

$$
\begin{aligned}
& \mathcal{E}_{0}(p)+\mathcal{E}_{k}(q)-\frac{\mathcal{E}_{k}(p+q)+\mathcal{E}_{k}(q-p)}{2}-\varepsilon_{0}(0) \\
& =\varepsilon_{1}(p)+\varepsilon_{1}(q)-\frac{\varepsilon_{1}(p+q)+\varepsilon_{1}(q-p)}{2}-\varepsilon_{1}(0) \\
& +\varepsilon_{2}(p)+\varepsilon_{2}(k-q)-\frac{\varepsilon_{2}(k-q-p)+\varepsilon_{2}(k-q+p)}{2}-\varepsilon_{2}(0)>0, \quad \text { a.e. } p \in \mathbb{T}^{3} .
\end{aligned}
$$

Our first non-perturbative result shows that under Hypothesis 5.1 the discrete spectrum of the fiber operators $h(k)$ under the variation of the quasi-momentum cannot be absorbed by the threshold, provided that $h(0)$ has eigenvalues below the bottom of its essential spectrum.

Theorem 5.3. Assume Hypothesis 5.1 Denote by $m(k), k \in \mathbb{T}^{3}$, the lower bound of the operator $h(k)$,

$$
m(k)=\inf \operatorname{spec}(h(k)), \quad k \in \mathbb{T}^{3} .
$$

If the Hamiltonian $h(0)$ does not have the discrete spectrum below the bottom of its essential spectrum, that is, $m(0)=\mathcal{E}_{\min }(0)$, assume, in addition, that the lower edge $m(0)$ of the spectrum of the operator $h(0)$ is an eigenvalue. Then

$$
\mathcal{E}_{\min }(0)-m(0)<\mathcal{E}_{\min }(k)-m(k), \quad k \in \mathbb{T}^{3}, k \neq 0 .
$$

Proof. Let $0 \neq f \in \operatorname{Ker}(h(0)-m(0) I)$ and hence

$$
\mathcal{E}_{0}(p) f(p)+(2 \pi)^{-\frac{3}{2}} \int_{\mathbb{T}^{3}} v(p-q) f(q) d q=m(0) f(p), \quad \text { a.e. } p \in \mathbb{T}^{3} .
$$

By hypothesis the one-particle dispersion relations are conditionally negative definite functions. Then, as it can easily be seen from the definition of the two-body dispersion 
relation, the function $\mathcal{E}_{0}(p)$ corresponding to the zero-value of the quasi-momentum $k$ is also conditionally negative definite. In particular, $\mathcal{E}_{0}(p)$ is an even functions and, hence, by Remark 3.8 without loss of generality one may assume that the function $|f(\cdot)|$ is even.

For $k \in \mathbb{T}^{3}$ we introduce the trial $L^{2}\left(\mathbb{T}^{3}\right)$-function

$$
f_{k}(p)=f(p-p(k)),
$$

where $p(k)$ denotes the minimum point of the function $\mathcal{E}_{k}(p)$, that is, $\mathcal{E}_{k}(p(k))=\mathcal{E}_{\min }(k)$ (if the minimum value of $\mathcal{E}_{k}(p)$ is attained in several points choose $p(k)$ as any one of them arbitrarily).

To prove $[5.2$ it is sufficient to establish the inequality

$$
\Gamma(k)=\left(\left[h(k)-\left(\mathcal{E}_{\min }(k)-\mathcal{E}_{\min }(0)+m(0)\right)\right] f_{k}, f_{k}\right)<0, \quad k \neq 0 .
$$

One gets

$$
\begin{aligned}
& \left(\left[h(k)-\left(\mathcal{E}_{\min }(k)-\mathcal{E}_{\min }(0)+m(0)\right)\right] f_{k}\right)(p) \\
& =\left[\mathcal{E}_{k}(p)-\left(\mathcal{E}_{\min }(k)-\mathcal{E}_{\min }(0)+m(0)\right)\right] f(p-p(k)) \\
& +(2 \pi)^{-\frac{3}{2}} \int_{\mathbb{T}^{3}} v(p-q) f(q-p(k)) d q \\
& =\left[\mathcal{E}_{k}(p)-\left(\mathcal{E}_{\min }(k)-\mathcal{E}_{\min }(0)+m(0)\right)\right] f(p-p(k)) \\
& +(2 \pi)^{-\frac{3}{2}} \int_{\mathbb{T}^{3}} v(p-p(k)-q) f(q) d q \\
& =\left[\mathcal{E}_{k}(p)-\mathcal{E}_{\min }(k)-\mathcal{E}_{0}(p-p(k))+\mathcal{E}_{\min }(0)\right] f(p-p(k)) .
\end{aligned}
$$

Using (5.4) one arrives at the representation

$$
\begin{gathered}
\Gamma(k)=-\int_{\mathbb{T}^{3}}\left(\varepsilon_{0}(p-p(k))-\mathcal{E}_{\min }(0)-\mathcal{E}_{k}(p)+\mathcal{E}_{\min }(k)\right)|f(p-p(k))|^{2} d p, \\
k \in \mathbb{T}^{3} .
\end{gathered}
$$

To check the basic inequality 5.3 we proceed as follows.

Making the change of variable $p \rightarrow-p+2 p(k)$ in (5.5) and using the fact that the functions $\mathcal{E}_{0}(p)$ and $|f(p)|$ are even, one obtains the representation

$$
\Gamma(k)=-\int_{\mathbb{T}^{3}}\left(\varepsilon_{0}(p-p(k))-\varepsilon_{\min }(0)-\varepsilon_{k}(-p+2 p(k))+\varepsilon_{\min }(k)\right)|f(p-p(k))|^{2} d p \text {. }
$$

Making again the change of variable $q \rightarrow p-p(k)$ in 5.5 and 5.6 and adding the results obtained we get

$$
\Gamma(k)=-\int_{\mathbb{T}^{3}} \mathcal{F}(k, p)|f(p)|^{2} d p,
$$

where

$$
\mathcal{F}(k, p)=\varepsilon_{0}(p)-\varepsilon_{\min }(0)+\varepsilon_{\min }(k)-\frac{\mathcal{E}_{k}(p+p(k))+\mathcal{E}_{k}(p(k)-p)}{2} .
$$

By Lemma 5.2 for any (fixed) $k \neq 0$, one concludes that $\mathcal{F}(k, p)>0$ for almost every $p \in \mathbb{T}^{3}$, proving the basic inequality $[5.3$ and the claim follows.

Our second non-perturbative result provides sufficient conditions for the discrete spectrum of the whole family of fiber Hamiltonians $h(k)$ with $k \neq 0$ to be non-empty. 
Theorem 5.4. Assume Hypothesis 5.1] Assume, in addition, that the operator $h(0)$ has either a threshold eigenvalue or a virtual level. Then, for all $k \in \mathbb{T}^{3} \backslash\{0\}$ the discrete spectrum of the fiber Hamiltonian $h(k)$ below the bottom $\mathcal{E}_{\min }(k)$ of its essential spectrum is a non-empty set.

Proof. The case where $h(0)$ has eigenvalues below the bottom of its essential spectrum has been already treated in Theorem 5.3 Assume, therefore, that the lower bound $m(0)$ of $h(0)$ coincides with the bottom of its essential spectrum, that is,

$$
m(0)=\mathcal{E}_{\min }(0)=\mathcal{E}_{0}(0) .
$$

If, under this hypothesis, $\varepsilon_{0}(0)$ is a (threshold) eigenvalue, the claim follows from Theorem 5.3

Assume, then, that $h(0)$ has a virtual level at the bottom of its essential spectrum. Therefore, the equation

$$
G\left(\mathcal{E}_{0}(0)\right) \psi=-\psi, \quad \psi \in C\left(\mathbb{T}^{3}\right),
$$

has a nontrivial solution $\psi \in C\left(\mathbb{T}^{3}\right)$. As in the proof of Theorem 5.3 without loss of generality one may assume that the function $|\psi(p)|$ is even. In particular, the equation

$$
\mathcal{E}_{0}(p) f(p)+(2 \pi)^{-\frac{3}{2}} \int_{\mathbb{T}^{3}} v(p-q) f(q) d q=m(0) f(p), \quad \text { a.e. } p \in \mathbb{T}^{3},
$$

has the $L^{1}\left(\mathbb{T}^{3}\right)$-solution (cf. Remark 3.7

$$
f(p)=\frac{\psi(p)}{\varepsilon_{0}(p)-\varepsilon_{0}(0)}
$$

such that the function $|f(\cdot)|$ is even.

Given $k \in \mathbb{T}^{3}$, introduce the sequence $\left\{f_{n, k}\right\}_{n=1}^{\infty}$ of $L^{2}\left(\mathbb{T}^{3}\right)$-functions

$$
f_{n, k}(p)=\frac{\psi(p-p(k))}{\mathcal{E}_{0}(p-p(k))-\mathcal{E}_{0}(0)+\frac{1}{n}} .
$$

By the dominated convergence Theorem the sequence $f_{n, k}$ converges in the space $L^{1}\left(\mathbb{T}^{3}\right)$ as $n \rightarrow \infty$ to the function $f_{k}$

$$
f_{k}(p)=f(p-p(k)), \quad p \in \mathbb{T}^{3},
$$

with $f(\cdot)$ a summable majorant.

Under Hypothesis 3.1 this means that the sequence of functions $\left[h(k)-\mathcal{E}_{\min }(k)\right] f_{k, n}$ converges in $L^{\infty}\left(\mathbb{T}^{3}\right)$-norm to the bounded function

$$
\begin{aligned}
& {\left[\mathcal{E}_{k}(p)-\mathcal{E}_{\min }(k)\right] f_{k}(p)+(2 \pi)^{-\frac{3}{3}} \int_{\mathbb{T}^{3}} v(p-q) f_{k}(q) d q } \\
= & {\left[\mathcal{E}_{k}(p)-\mathcal{E}_{\min }(k)-\mathcal{E}_{0}(p-p(k))+\mathcal{E}_{0}(0)\right] f(p-p(k)), }
\end{aligned}
$$

where we used (5.7), 5.8) and the representations

$$
\begin{aligned}
& \left(\left[h(k)-\mathcal{E}_{\min }(k)\right] f_{k, n}\right)(p) \\
& =\left[\mathcal{E}_{k}(p)-\mathcal{E}_{\min }(k)\right] f_{k, n}(p)+(2 \pi)^{-\frac{3}{3}} \int_{\mathbb{T}^{3}} v(p-q) f_{k, n}(q) d q .
\end{aligned}
$$

In particular, one concludes that the limit

$$
\Gamma(k)=\lim _{n \rightarrow \infty}\left(\left[h(k)-\mathcal{E}_{\min }(k)\right] f_{n, k}, f_{n, k}\right), \quad k \in \mathbb{T}^{3},
$$


exists and is finite and, moreover,

$$
\Gamma(k)=-\int_{\mathbb{T}^{3}} \frac{\mathcal{E}_{0}(p-p(k))-\mathcal{E}_{0}(0)-\mathcal{E}_{k}(p)+\mathcal{E}_{\min }(k)}{\left(\mathcal{E}_{0}(p-p(k))-\mathcal{E}_{0}(0)\right)^{2}}|\psi(p-p(k))|^{2} d p, \quad k \in \mathbb{T}^{3} .
$$

Next, exactly as it has been done in the proof of Theorem 5.3 one checks the inequality

$$
\Gamma(k)<0, \quad k \neq 0 .
$$

It follows from (5.9) that there exists an $n_{0} \in \mathbb{N}$ such that

$$
\left(\left[h(k)-\mathcal{E}_{\min }(k)\right] f_{n_{0}, k}, f_{n_{0}, k}\right)<0, \quad k \neq 0
$$

proving the existence of the discrete spectrum of $h(k)$ below its essential spectrum for $k \neq 0$. The proof is complete.

Remark 5.5. If the operator $h(0)$ has a multiple threshold eigenvalue, then using the same strategy of proof one concludes that the variational estimate

$$
\left(\left[h(k)-\mathcal{E}_{\min }(k)\right] f_{k}, f_{k}\right)<0, \quad k \neq 0,
$$

with

$$
f_{k}(p)=f(p-p(k)),
$$

holds for any $0 \neq f \in \operatorname{Ker}\left(h(0)-\mathcal{E}_{\min }(0) I\right)$. Therefore, estimate (5.10) holds for all functions $f_{k}(\cdot)$ of the form (5.11) from a subspace of dimensionality

$$
d=\operatorname{dim} \operatorname{Ker}\left(h(0)-\mathcal{E}_{\min }(0) I\right) .
$$

This means that the number $N$ of eigenvalues of $h(k)$ with $k \neq 0$ (counting multiplicity) below the bottom of the essential spectrum admits the estimate

$$
N \geq \max \{1, d\} \text {. }
$$

Remark 5.6. The width $w(k)$ of the essential spectrum band of the Hamiltonians $h(k)$,

$$
w(k)=\varepsilon_{\max }(k)-\varepsilon_{\min }(k)
$$

may vanish for some values of the quasi-momentum $k \in \mathbb{T}^{3}$. Therefore, the fiber Hamiltonians $h(k)$ may have an infinite discrete spectrum for some values of the quasi-momentum $k$ even if the spectrum of $h(0)$ is essential. For instance, consider two (identical) particles with the one-particle dispersion relations of the form

$$
\varepsilon_{1}(p)=\varepsilon_{2}(p)=\sum_{i=1}^{3}\left(1-\cos p_{i}\right), \quad p \in \mathbb{T}^{3} .
$$

Then if $k_{0}=(\pi, \pi, \pi) \in \mathbb{T}^{3}$ we have the "strong degeneration" of the two-particle dispersion relation:

$$
\mathcal{E}_{k_{0}}(p)=\varepsilon_{1}(p)+\varepsilon_{2}\left(k_{0}-p\right)=6 \quad \text { holds for all } p=\left(p_{1}, p_{2}, p_{3}\right) \in \mathbb{T}^{3} .
$$

Therefore, in this case, the fiber Hamiltonian $h\left(k_{0}\right)$ associated with the system of two particles with dispersion relations (5.12) interacting via a fast decreasing potential $v$ with infinitely many negative eigenvalues has an infinite discrete spectrum below the bottom of its essential spectrum. (In this particular example the essential spectrum of $h\left(k_{0}\right)$ is a one-point set, namely, $\left.\operatorname{spec}_{e s s}\left(h\left(k_{0}\right)\right)=\{6\}\right)$. 
It is also worth mentioning that even a partial degeneracy of the two-particle dispersion relation $\mathcal{E}_{k}(\cdot)$ for some values of the quasi-momentum $k \neq 0$ may generate a "rich" infinite discrete spectrum of the Hamiltonian $h(k)$ outside the band $\left[\mathcal{E}_{\min }(k), \mathcal{E}_{\max }(k)\right]$.

\section{ApPendix A. Proof of Proposition 3.9}

Assume without loss of generality that $\varepsilon(0)=0$.

"Only If Part." Let $f \in L^{2}\left(\mathbb{T}^{3}\right)$ be an eigenfunction of the operator $h(0)$ associated with a zero eigenvalue, that is,

$$
-\varepsilon(p) f(p)=(2 \pi)^{-\frac{3}{2}} \int_{\mathbb{T}^{3}} v(p-q) f(q) d q, \quad \text { a.e. } p \in \mathbb{T}^{3} .
$$

The same argument as in the proof of Lemma 3.2 shows that the equivalence class associated with the function $f$ has a representative $\tilde{f}$ such that the function

$$
\psi(p)=\varepsilon(p) \tilde{f}(p)
$$

is Hölder continuous, $\psi \in \mathcal{B}(\kappa)$. Hence the representative $\tilde{f}$ is continuous away from the origin and since from Hypothesis 3.1 it follows that $\liminf _{p \rightarrow 0} \varepsilon(p)|p|^{-2}>0$, the following asymptotic representation

$$
\tilde{f}(p)=\frac{\psi(0)}{\varepsilon(p)}+\mathcal{O}\left(|p|^{-2+\kappa}\right), \quad p \rightarrow 0,
$$

holds. Since $\tilde{f} \in L^{2}\left(\mathbb{T}^{3}\right)$ and $\kappa>\frac{1}{2}$, the Hölder continuous function $\psi$ must vanish at the origin, that is, $\psi(0)=0$.

Comparing A.1 and A.2 one concludes that -1 is an eigenvalue of the operator $G(\varepsilon(0))$ on $C\left(\mathbb{T}^{3}\right)$ associated with the eigenfunction $\psi$ with $\psi(0)=0$.

"If Part." Assume that the operator $G(\varepsilon(0))$ has an eigenfunction $\psi$ associated with the eigenvalue $\lambda=-1$,

$$
G(\varepsilon(0)) \psi=-\psi
$$

such that $\psi(0)=0$. Following the strategy of proof of Lemma 3.2 one gets that $\psi \in \mathcal{B}(\kappa)$. Introduce the function

$$
f(p)=\frac{\psi(p)}{\varepsilon(p)}, \quad p \neq 0
$$

Clearly, an argument as above shows that the following asymptotic representation

$$
f(p)=\mathcal{O}\left(|p|^{-2+\kappa}\right), \quad p \rightarrow 0,
$$

holds. Since $\kappa>\frac{1}{2}$, one proves that $f \in L^{2}\left(\mathbb{T}^{3}\right)$ and then A.3 means that the operator $h(0)$ has a nontrivial kernel, completing the proof.

\section{ApPendix B. Coexistence of A threshold eigenvalue AND A ViRTual LEVEL}

The main goal of this Appendix is to show by an explicit example that the Case IV is not empty.

Example B.1. Let $\hat{h}_{\lambda, \mu}, \lambda, \mu \in \mathbb{R}$, be the discrete Schrödinger operator of the form

$$
\hat{h}_{\lambda, \mu}=-\Delta+\hat{v}_{\lambda, \mu}
$$


where $\Delta$ is the discrete Laplacian from Example 2.1 and

$$
\hat{v}_{\lambda, \mu}(s)= \begin{cases}\mu, & s=0 \\ \frac{\lambda}{2}, & |s|=1 \\ 0, & \text { otherwise. }\end{cases}
$$

The Fourier transform of the interaction can be explicitly computed

$$
v(p)=\frac{1}{(2 \pi)^{\frac{3}{2}}}\left(\mu+\lambda \sum_{i=1}^{3} \cos p_{i}\right)
$$

and, hence, for the Birman-Schwinger kernel one gets the representation

$$
G(p, q ; 0)=\frac{1}{(2 \pi)^{3}} \frac{\mu+\lambda \sum_{i=1}^{3} \cos \left(p_{i}-q_{i}\right)}{\varepsilon(q)}, \quad p, q \in \mathbb{T}^{3},
$$

where $\varepsilon(q)$ is given by (2.3) and we have used the equality $\varepsilon(0)=0$.

Introduce the notations

$$
\begin{aligned}
& a=\frac{1}{(2 \pi)^{3}} \int_{\mathbb{T}^{3}} \frac{d q}{\varepsilon(q)}, \quad c=\frac{1}{(2 \pi)^{3}} \int_{\mathbb{T}^{3}} \frac{\cos q_{i} d q}{\varepsilon(q)}, \quad i=1,2,3, \\
& s=\frac{1}{(2 \pi)^{3}} \int_{\mathbb{T}^{3}} \frac{\sin ^{2} q_{i} d q}{\varepsilon(q)}, \quad b=\frac{1}{(2 \pi)^{3}} \int_{\mathbb{T}^{3}} \frac{\cos ^{2} q_{i} d q}{\varepsilon(q)}, \quad i=1,2,3, \\
& d=\frac{1}{(2 \pi)^{3}} \int_{\mathbb{T}^{3}} \frac{\cos q_{i} \cos q_{j} d q}{\varepsilon(q)}, \\
& i, j=1,2,3, i \neq j .
\end{aligned}
$$

We remark that since the function $\varepsilon(q)=\varepsilon\left(q_{1}, q_{2}, q_{3}\right)$ is invariant with respect to the permutations of its arguments $q_{1}, q_{2}$ and $q_{3}$, the integrals $c, s, b, d$ above do not depend on the particular choice of the indices $i, j$. A simple computation shows that the following relations

$$
\begin{aligned}
& a-c=\frac{1}{6}, \\
& b+2 d=3 c, \\
& a=b+s \quad \text { and } \quad s=\frac{1}{6}-\frac{2}{3}(b-d)
\end{aligned}
$$

hold.

Lemma B.2. $a>\frac{11}{51}$. In particular, $c>0$.

Proof. We start with the representation

$$
\begin{aligned}
\int_{-\pi}^{\pi} \frac{d q}{A-\cos q} & =\int_{-\frac{\pi}{4}}^{\frac{\pi}{4}} \frac{d q}{A-\cos q}+\int_{-\frac{\pi}{4}}^{\frac{\pi}{4}} \frac{d q}{A+\cos q} \\
& +\int_{-\frac{\pi}{4}}^{\frac{\pi}{4}} \frac{d q}{A-\sin q}+\int_{-\frac{\pi}{4}}^{\frac{\pi}{4}} \frac{d q}{A+\sin q}, \quad|A|>1,
\end{aligned}
$$

which yields

$$
\begin{aligned}
a & =\frac{1}{(2 \pi)^{3}} \int_{\mathbb{T}^{3}} \frac{d q}{\varepsilon(q)}=\frac{1}{2(2 \pi)^{3}} \int_{\mathbb{T}^{3}} \frac{d q}{3-\cos q_{1}-\cos q_{2}-\cos q_{3}} \\
& =\frac{1}{2(2 \pi)^{3}} \int_{\mathbb{T}^{2}} d q_{1} d q_{2} \int_{-\frac{\pi}{4}}^{\frac{\pi}{4}} d q_{3} f\left(q_{1}, q_{2}, q_{3}\right),
\end{aligned}
$$


where

$$
\begin{aligned}
f\left(q_{1}, q_{2}, q_{3}\right) & =\frac{1}{3-\cos q_{1}-\cos q_{2}-\cos q_{3}}+\frac{1}{3-\cos q_{1}-\cos q_{2}+\cos q_{3}} \\
& +\frac{1}{3-\cos q_{1}-\cos q_{2}-\sin q_{3}}+\frac{1}{3-\cos q_{1}-\cos q_{2}+\sin q_{3}}
\end{aligned}
$$

Note that the function $f$ is well defined on $(-\pi, \pi]^{3} \backslash\{0\}$.

One easily checks that for fixed $q_{1}, q_{2}$, the function $f\left(q_{1}, q_{2}, q_{3}\right)$ as a function of the argument $q_{3}, q_{3} \in\left[-\frac{\pi}{4}, \frac{\pi}{4}\right]$, attains its minimal value at the end points of the interval $\left[-\frac{\pi}{4}, \frac{\pi}{4}\right]$ and hence

$$
\begin{gathered}
f\left(q_{1}, q_{2}, q_{3}\right)>\frac{2}{3-\cos q_{1}-\cos q_{2}-\frac{\sqrt{2}}{2}}+\frac{2}{3-\cos q_{1}-\cos q_{2}+\frac{\sqrt{2}}{2}}, \\
q_{3} \in\left(-\frac{\pi}{4}, \frac{\pi}{4}\right) .
\end{gathered}
$$

Combining (B.6) and B.7 proves the inequality

$$
a>\frac{1}{(2 \pi)^{3}} \frac{\pi}{2} \int_{\mathbb{T}^{2}} d q_{1} d q_{2}\left(\frac{1}{3-\frac{\sqrt{2}}{2}-\cos q_{1}-\cos q_{2}}+\frac{1}{3+\frac{\sqrt{2}}{2}-\cos q_{1}-\cos q_{2}}\right) .
$$

Applying the trick B.5 two more times (first by getting rid of the variable $q_{2}$ and then of $q_{1}$ ) one arrives at the estimate

$$
\begin{aligned}
a & >\frac{1}{(2 \pi)^{3}}\left(\frac{\pi}{2}\right)^{2} \int_{\mathbb{T}} d q_{1}\left(\frac{2}{3-2 \frac{\sqrt{2}}{2}-\cos q_{1}}+\frac{4}{3-\cos q_{1}}+\frac{2}{3+2 \frac{\sqrt{2}}{2}-\cos q_{1}}\right) \\
& >\frac{1}{(2 \pi)^{3}}\left(\frac{\pi}{2}\right)^{3}\left[\frac{4}{3-3 \frac{\sqrt{2}}{2}}+\frac{12}{3-\frac{\sqrt{2}}{2}}+\frac{12}{3+\frac{\sqrt{2}}{2}}+\frac{4}{3+3 \frac{\sqrt{2}}{2}}\right]=\frac{11}{51},
\end{aligned}
$$

completing the proof.

Corollary B.3. The set

$$
\Lambda=\left\{\frac{1}{s}, \frac{1}{b-d}\right\} \backslash\left\{\frac{2 a}{c}\right\}
$$

is nonempty.

Proof. Assume to the contrary that $\Lambda=\emptyset$, that is,

$$
\frac{1}{s}=\frac{1}{b-d}=\frac{2 a}{c} \text {. }
$$

Solving (B.3, B.4 and (B.8) simultaneously in particular yields

$$
a=\frac{5}{24}<\frac{11}{51}
$$

which is impossible due to Lemma.2

Theorem B.4. Assume that $-\lambda \in \Lambda$ and

$$
\mu=-\frac{1+3 \lambda c}{a+\frac{\lambda c}{2}}
$$

then the Hamiltonian $h_{\lambda, \mu}$ has both a virtual level and a threshold eigenvalue. 
Proof. In accordance with Proposition 3.9 one needs to show that the integral operator $G(0)$ given by $\mathrm{B} .1$ on the Banach space $C\left(\mathbb{T}^{3}\right)$ has two eigenfunctions, $\psi$ and $\varphi$ associated with an eigenvalue -1 :

$$
G(0) \psi=-\psi \quad \text { and } \quad G(0) \varphi=-\varphi
$$

such that

$$
\psi(0) \neq 0 \quad \text { and } \quad \varphi(0)=0 .
$$

The space of all odd (resp. even) functions $C_{o}\left(\mathbb{T}^{3}\right)$ (resp. $C_{e}\left(\mathbb{T}^{3}\right)$ ) is an invariant subspace for the integral operator $G(0)$. The restrictions $G_{o}$ (respectively $G_{e}$ ) of $G(0)$ on the subspace $C_{o}\left(\mathbb{T}^{3}\right)$ (respectively $C_{o}\left(\mathbb{T}^{3}\right)$ ) have the kernel functions

$$
\begin{aligned}
& G_{o}(p, q)=\frac{\lambda}{(2 \pi)^{3}} \sum_{i=1}^{3} \frac{\sin p_{i} \sin q_{i}}{\varepsilon(q)}, \\
& G_{e}(p, q)=\frac{1}{(2 \pi)^{3}} \frac{\mu+\lambda \sum_{i=1}^{3} \cos p_{i} \cos q_{i}}{\varepsilon(q)} .
\end{aligned}
$$

The matrix of the restriction $\left.G_{o}\right|_{S}$ of $G_{O}$ onto its three-dimensional invariant subspaces $S \subset C_{o}\left(\mathbb{T}^{3}\right)$ spanned by the functions $\sin p_{1}, \sin p_{2}$, and $\sin p_{3}$ in the basis $e_{i}=\sin p_{i}$, $i=1,2,3$, is a diagonal matrix of the form

$$
\left.G_{o}\right|_{S}=\left(\begin{array}{ccc}
\lambda s & 0 & 0 \\
0 & \lambda s & 0 \\
0 & 0 & \lambda s
\end{array}\right)
$$

while the matrix of the restriction $\left.G_{e}\right|_{C}$ of $G_{e}$ onto its four-dimensional invariant subspaces $C \subset C_{e}\left(\mathbb{T}^{3}\right)$ spanned by the functions $1, \cos p_{1}, \cos p_{2}$, and $\cos p_{3}$ in the basis $f_{i}=\cos p_{i}$, $i=1,2,3, f_{4}=1$ is given by

$$
\left.G_{e}\right|_{C}=\left(\begin{array}{cccc}
\lambda b & \lambda d & \lambda d & \lambda c \\
\lambda d & \lambda b & \lambda d & \lambda c \\
\lambda d & \lambda d & \lambda b & \lambda c \\
\mu c & \mu c & \mu c & \mu a
\end{array}\right) .
$$

From (B.10) it follows that if $\lambda, \mu$ and $\gamma$ satisfy the relations

$$
\lambda(b+2 d+c \gamma)=-1
$$

then

$$
\mu(3 c+a \gamma)=-\gamma
$$

$$
\left(\begin{array}{cccc}
\lambda b & \lambda d & \lambda d & \lambda c \\
\lambda d & \lambda b & \lambda d & \lambda c \\
\lambda d & \lambda d & \lambda b & \lambda c \\
\mu c & \mu c & \mu c & \mu a
\end{array}\right)\left(\begin{array}{l}
1 \\
1 \\
1 \\
\gamma
\end{array}\right)=\left(\begin{array}{l}
1 \\
1 \\
1 \\
\gamma
\end{array}\right)
$$

Given $\lambda \in \mathbb{R}, \lambda \neq-\frac{2 a}{c}$, solving equations $\mathbb{B}$.11 and B.12 with respect to $\mu$ and $\gamma$ yields

$$
\begin{aligned}
& \gamma(\lambda)=-\frac{1}{\lambda c}-3 \\
& \mu(\lambda)=-\frac{\gamma(\lambda)}{3 c+a \gamma(\lambda)}=\frac{1+3 \lambda c}{3 \lambda c^{2}-3 \lambda a c-a}=-\frac{1+3 \lambda c}{a+\frac{\lambda c}{2}}
\end{aligned}
$$


where we used (B.2) and B.3. Therefore, if $\lambda \neq-\frac{2 a}{c}$ and $\mu=\mu(\lambda)$ satisfies (B.14) the operator $G(0)$ has an eigenfunction $\psi(p)=\gamma(\lambda)+\sum_{i=1}^{3} \cos p_{i}$ and, moreover,

$$
\psi(0)=\left.\left(\gamma(\lambda)+\sum_{i=1}^{3} \cos p_{i}\right)\right|_{p_{1}=p_{2}=p_{3}=0} \neq 0
$$

as it follows from B.13. Thus, the Hamiltonian $h_{\lambda, \mu(\lambda)}$ has a virtual level.

Next, from the matrix representation B.9 for $\left.G_{o}\right|_{S}$ one gets that if

$$
\lambda s=-1
$$

then for any $\mu \in \mathbb{R}$ the operator $G_{o}$ has a three-dimensional eigensubspace spanned by the functions $\sin p_{i}, i=1,2,3$, associated with an eigenvalue -1 of multiplicity three. In particular, $G(0) \varphi=-\varphi$ with $\varphi(p)=\sin p_{1}$ and hence $\varphi(0)=0$.

Similarly (cf. (B.10), if

$$
\lambda(b-d)=-1
$$

then for any $\mu \in \mathbb{R}$ the operator $\left.G_{e}\right|_{C}$ has two linearly independent eigenfunctions $\cos p_{1}-$ $\cos p_{2}$ and $\cos p_{1}-\cos p_{3}$ associated with an eigenvalue -1 of multiplicity two. In particular, $G \varphi=-\varphi$ with $\varphi(p)=\cos p_{1}-\cos p_{2}$, and hence $\varphi(0)=0$.

Therefore, if $\lambda=-\frac{1}{s}$ or $\lambda=-\frac{1}{b-d}$, then for any $\mu \in \mathbb{R}$ the operator $h_{\lambda, \mu}$ has an eigenvalue at the bottom of its (absolutely) continuous spectrum.

Taking $-\lambda \in \Lambda=\left\{\frac{1}{s}, \frac{1}{b-d}\right\} \backslash\left\{\frac{2 a}{c}\right\}$ (which is nonempty by Corollary B.3) and $\mu=$ $\mu(\lambda)=-\frac{1+3 \lambda c}{a+\frac{\lambda c}{2}}$ one proves the coexistence of a virtual level and a threshold eigenvalue for the Hamiltonian $h_{\lambda, \mu(\lambda)}$.

Remark B.5. We were not able to find out whether the set $\Lambda$ is a one-or a two-point set and hence we cannot explicitly compute the multiplicity of the zero-energy eigenvalue (more information about numerical values of the integrals $a, b, c$, and $d$ is needed). However, if $\Lambda$ contains two elements, then the Hamiltonian $\hat{h}_{\lambda,-\frac{1+3 \lambda c}{a+\frac{\lambda c}{2}}}$ has a virtual level

and a threshold eigenvalue of multiplicity two or three depending on the choice of $-\lambda \in$ $\Lambda\left(\lambda=-\frac{1}{b-d}\right.$ or $\lambda=-\frac{1}{s}$ respectively).

If $|\Lambda|=1$, it might happen that the Hamiltonian $\hat{h}_{\lambda,-\frac{1+3 \lambda c}{a+\frac{\lambda c}{2}}},-\lambda \in \Lambda$, has a virtual level and a threshold eigenvalue of multiplicity two, three or even five depending on which of the cases

(i) $\frac{c}{2 a}=s \neq b-d$;

(ii) $\frac{c}{2 a}=b-d \neq s$;

(iii) $\frac{c}{2 a} \neq s=b-d$; takes place respectively.

Acknowledgments K. A. Makarov thanks F. Gesztesy and V. Kostrykin for useful discussions. He is also indebted to the Institute of Applied Mathematics of the University Bonn for its kind hospitality during his stay in the summer 2003. This work was also partially supported by the DFG 436 USB 113/4 Project and the Fundamental Science Foundation of Uzbekistan. S. N. Lakaev and Z. I. Muminov gratefully acknowledge the hospitality of the Institute of Applied Mathematics of the University Bonn. 


\section{REFERENCES}

[1] Albeverio, S., Gesztesy F., and Høegh-Krohn R.: The low energy expansion in non-relativistic scattering theory. Ann. Inst. H. Poincaré Sect. A (N.S.) 37, 1-28 (1982).

[2] Albeverio S., Høegh-Krohn R., and Wu T. T.: A class of exactly solvable three-body quantum mechanical problems and universal low energy behavior. Phys. Lett. A 83, 105-109 (1971).

[3] Albeverio S., Gesztesy F., Høegh-Krohn R., and Holden H.: Solvable Models in Quantum Mechanics. Springer-Verlag, New York, 1988; 2nd ed. (with an appendix by P. Exner), Chehea, AMS, 2004.

[4] Albeverio S., Lakaev S. N., and Muminov Z. I.: Schrödinger operators on lattices. The Efimov effect and discrete spectrum asymptotics. Ann. Henri Poincaré. 5, 743-772 (2004).

[5] Berg C., Christensen J. P. R, and Ressel P.: Harmonic analysis on semigroups. Theory of positive definite and related functions. Graduate Texts in Mathematics, Springer-Verlag, New York, 1984. 289 pp.

[6] Carmona R. and Lacroix J.: Spectral theory of random Schrödinger operators. Probability and its Applications, 1990, Birkhäuser Boston.

[7] Jensen A. and Kato T.: Spectral properties of Schrödinger operators and time-decay of the wave functions. Duke Math. J. 46. 583-611 (1979).

[8] Faria da Veiga P. A., Ioriatti L., and O'Carroll M.: Energy-momentum spectrum of some two-particle lattice Schrödinger Hamiltonians. Phys. Rev. E (3) 66, 016130, 9 pp. (2002).

[9] Graf G. M. and Schenker D.: 2-magnon scattering in the Heisenberg model. Ann. Inst. H. Poincaré Phys. Théor. 67, 91-107 (1997).

[10] Klaus M. and Simon B.: Coupling constants thresholds in non-relativistic quantum mechanics. I. Short range two body case. Ann. Phys. 130, 251-281 (1980).

[11] Kondratiev Yu. G. and R. A. Minlos R. A.: One-particle subspaces in the stochastic $X Y$ model. J. Statist. Phys. 87, 613-642 (1997).

[12] Kostrykin V. and Schrader R.: Cluster properties of one particle Scrödinger operators. II. Rev. Math. Phys. 10, 627-682 (1998).

[13] Lakaev S. N.: The Efimov effect in a system of three identical quantum particles. Funct. Anal. Appl. 27, 166-175 (1993).

[14] Lakaev S. N.: Discrete spectrum and resonances of the one-dimensional Schrödinger operator for small coupling constants. Teoret. Mat. Fiz. 44, 381-386 (1980).

[15] Mattis D. C.: The few-body problem on a lattice. Rev. Modern Phys. 58, 361-379 (1986).

[16] Minlos R. A. and Suhov Y. M.: On the spectrum of the generator of an infinite system of interacting diffusions. Comm. Math. Phys. 206, 463-489 (1999).

[17] Mogilner A.: Hamiltonians in solid state physics as multi-particle discrete Schrödinger operators: Problems and results. Advances in Soviet Mathematics 5, 139-194 (1991).

[18] Ovchinnikov Yu. N. and Sigal I. M.: Number of bound states of three-particle systems and Efimov's effect. Ann. Physics 123, 274-295 (1989).

[19] Rauch J.: Perturbation theory for eigenvalues and resonances of Schrödinger Hamiltonians. J. Funct. Anal. 35, 304-315 (1980).

[20] Reed M. and Simon B.: Methods of modern mathematical physics. III: Scattering theory, Academic Press, New York, 1979.

[21] Reed M. and Simon B.: Methods of modern mathematical physics. VI: Analysis of Operators, Academic Press, New York, 1979.

[22] Simon B.: Large time behavior of the $L^{p}$ norm of Schrödinger Semigroups. J. Funct. Anal. 40, 66-83 (1981).

[23] Sobolev A. V.: The Efimov effect. Discrete spectrum asymptotics. Commun. Math. Phys. 156, 127-168 (1993).

[24] Tamura H.: The Efimov effect of three-body Schrödinger operators. J. Funct. Anal. 95, 433-459 (1991).

[25] Tamura H.: The Efimov effect of three-body Schrödinger operators: Asymptotics for the number of negative eigenvalues. Nagoya Math. J. 130, 55-83(1993).

[26] Yafaev D. R.: Scattering theory: Some old and new problems, Lecture Notes in Mathematics, 1735. Springer-Verlag, Berlin, 2000, 169 pp.

[27] Yafaev D. R.: On the theory of the discrete spectrum of the three-particle Schrödinger operator. Math. USSR-Sb. 23, 535-559 (1974).

[28] Yafaev D. R.: The virtual level of the Schrödinger equation. J. Soviet. Math., 11, 501-510 (1979).

[29] Zhizhina E. A.: Two-particle spectrum of the generator for stochastic model of planar rotators at high temperatures. J. Statist. Phys. 91, 343-368 (1998). 
${ }^{1}$ Institut Für Angewandte Mathematik, Universität Bonn (Germany)

E-mail address: albeverio@uni.bonn.de

2 SFB 611, Bonn, BiBoS, Bielefeld - Bonn (GERMANy)

${ }^{3}$ CERFIM, LOCARNO AND USI (SWITZERLAND)

${ }^{4}$ SAMARKAND Division of ACADEMY OF SCIENCES OF UZBEKISTAN (UZBEKISTAN)

E-mail address: lakaev@wiener.iam.uni-bonn.de

${ }^{5}$ Department of Mathematics, University of Missouri, Columbia, MO, (USA)

E-mail address: makarov@math.missouri.edu

${ }^{6}$ SAMARKAND STATE UNIVERSity, SAMARKAND (UZBEKISTAN)

E-mail address: zimuminov@mail.ru 